\title{
Outcome for sinonasal malignancies: a population-based survey
}

\author{
Anna Hafström ${ }^{1,2}$ - Johanna Sjövall, ${ }^{1,2}$. Simon S. Persson ${ }^{1,2} \cdot$ Johan S. Nilsson $^{1,2} \cdot$ Christer Svensson $^{1,2}$. \\ Eva Brun ${ }^{2,3}$ (1) $\cdot$ Lennart Greiff ${ }^{1,2}$ (B)
}

Received: 15 June 2021 / Accepted: 23 August 2021 / Published online: 12 September 2021

(c) The Author(s) 2021, corrected publication 2022

\begin{abstract}
Purpose Sinonasal malignancies (SNM) represent a rare and complex group of cancers that includes a wide range of histopathological subtypes. Data from population-based cohorts are scarce but warranted as a basis for randomized controlled treatment trials (RCTs). Our aim was to assess overall and histology subset-specific outcomes for SNM patients treated at a tertiary referral centre.

Methods A retrospective, population-based, consecutive cohort of patients with SNMs diagnosed from 2001 through 2019 was examined. Outcome was analysed in relation to age, gender, site, stage, histopathology, and treatment.

Results Two-hundred and twenty-six patients were identified, whereof $61 \%$ presented with stage IV disease. $80 \%$ completed treatment with curative intent, which comprised surgery with neoadjuvant (29\%) or adjuvant (37\%) radiotherapy, monotherapy with surgery (22\%), definitive chemoradiotherapy (7\%), or radiotherapy (5\%). Median follow-up was 106 months. The 5- and 10-year overall survival rates were 57\% and 35\%, respectively. Median overall survival was 76 months (esthesioneuroblastoma: 147 months; adenocarcinoma: 117; salivary carcinoma: 88; mucosal melanoma: 69; squamous cell carcinoma: 51, undifferentiated carcinoma: 42; neuroendocrine carcinoma: 9; and NUT-carcinoma 5). The 5- and 10-year disease-free survival rates were $63 \%$ and $54 \%$, respectively, and disease-specific survival $83 \%$ and $66 \%$. Increasing age, stage IVB, melanoma histopathology, and treatment with definitive chemoradiotherapy emerged as significant independent prognostic risk factors for disease-specific mortality $(p \leq 0.001)$.

Conclusion The results indicate a seemingly good outcome in comparison to previous reports, particularly for mucosal melanoma, adenocarcinoma, and undifferentiated carcinoma. The study provides additional background for future RCTs focusing on histology subset-specific treatment for SNM.
\end{abstract}

Keywords Head and neck cancer - Sinonasal cancer · Mucosal melanoma - Adenocarcinoma $\cdot$ Multimodal cancer therapy . Head and neck surgery

\section{Introduction}

Sinonasal malignancies (SNM) represent a rare and complex group of cancers that characteristically includes a wide range of histopathological subtypes [1]. The treatment comprises surgery and radiotherapy (RT) with or without

Anna Hafström

anna.hafstrom@skane.se

1 Department of ORL, Head \& Neck Surgery, Skåne University Hospital, 22185 Lund, Sweden

2 Department of Clinical Sciences, Lund University, Lund, Sweden

3 Department of Oncology, Skåne University Hospital, Lund, Sweden chemotherapy (CT) [2], and 5-year overall survival (OS) ranges from 20 to 50\% [3]. With few exceptions [4-6], there is limited information on characteristics and fate of SNM derived from population-based cohorts. Arguably, such information is imperative to accurately design future randomized controlled trials (RCTs) and, thereby, to evaluate treatments in relation to specific SNM subsets.

The three Nordic studies by Thorup et al., Koivunen et al., and Filtenborg et al. utilize population-based cancer registries and aim to identify and study all cases in a defined population [4-6]. The principal drawback of such studies is a lack of detailed information, which can only be obtained by analysing patient records. Furthermore, as indicated by Filtenborg et al., the case detection rate may vary, e.g., as reflected by an $8 \%$ discrepancy between the Danish Cancer 
Registry and the Danish Head and Neck Cancer Group for SNM [6]. Another disadvantage of registry-based studies is that inclusion of histological subtypes may not be uniform, and that treatment regimen may vary between contributing centres [2, 4-7]. Taken together, this highlights a need for population-based studies that include detailed crosschecking with patient records.

In this study involving consecutive patients in a population-based cohort from the Southern Swedish Health Care Region treated at one tertiary academic referral centre, we aimed to analyse characteristics and fate (survival) of SNM based on clinical information (not registry data). Accordingly, overall survival (OS), disease-free survival (DFS), and disease-specific survival (DSS) were analysed in relation to age, gender, site, stage, histopathology, and treatment.

\section{Methods}

\section{Study design and patients}

The study was of a retrospective design and involved consecutive patients with SNM from the population-based 1.9 million cohort of inhabitants of the Southern Swedish Health Care Region. These patients were either diagnosed at Skåne University Hospital, Lund, or referred to us from the five sub-regional hospitals, which reflects that treatment of all patients with SNM in the region is centralized to Skåne University Hospital. All patients from the Southern Swedish Health Care Region diagnosed with a primary SNM between January 1, 2001 and December 31, 2019 were identified using ICD-10 codes C300 to C318 and crosschecked against the regional multidisciplinary tumour (MDT) board registry. Ethical approval was granted by the regional Ethics Review Board (2018/745). Patient records were scrutinized and data on age, gender, subsite, stage, and histopathology were collected as were diagnosis date and treatment. Assessment of histological margin status is difficult in SNM, especially retrospectively, and was not included in the analysis. Demographics and survival outcome were analysed. Patients with earlier sinonasal cancer were excluded from the study.

\section{Staging}

If applicable, patients were restaged according to the 8th Edition of the Union for International Cancer Control (UICC) TNM Classification of Malignant Tumors. Clinical information, including radiological findings, were obtained and used for restaging. Data were retrieved from the pathology reports and the following histopathologies were included: squamous cell carcinoma (SCC), sinonasal mucosal melanoma (SNMM), adenocarcinoma, salivary carcinoma, esthesioneuroblastoma (ENB), sinonasal undifferentiated carcinoma (SNUC), sinonasal neuroendocrine carcinoma (SNEC), and NUT carcinoma.

\section{Treatment and follow-up}

Data on treatment intent as well as administered treatment were collected with date and type of surgical resection as well as dates and doses of administered RT with or without concomitant chemotherapy $(\mathrm{RT} \pm \mathrm{CT})$. The therapy was categorized into the following groups: definitive $\mathrm{RT} \pm \mathrm{CT}$, surgery alone, surgery with neoadjuvant or adjuvant $\mathrm{RT} \pm \mathrm{CT}$. Treatment was also categorized into monotherapy or multimodality regimes that included surgery with either neoadjuvant or adjuvant $\mathrm{RT} \pm \mathrm{CT}$. RT was administered 5 days per week in daily doses of 1.8-3 Gray (Gy) per fraction once or twice daily. Follow-up was also recorded, including date and localization of any recurrence, date and cause of death, and date of last follow-up. Patients had clinical follow-up controls every 3rd-6th month either up to 5-year post-treatment, until December 31, 2020, or death.

\section{Analysis}

Median follow-up time was calculated from end of treatment to last follow-up or death. Disease free survival (DFS) was calculated as the time from end of primary treatment to the date of an event. Disease-specific survival (DSS) and overall survival (OS) were measured from date of diagnosis to date of death. Surviving patients still recurrence-free and/or alive at their last follow-up were censored on that date. Standard Kaplan-Meier estimates of DFS, DSS, and OS distributions were computed using SPSS 25.0. Differences between survival curves were assessed by the log-rank test. Analysis of prognostic factors was performed using Cox proportional hazard analyses.

\section{Results}

Two-hundred and forty-three SNM patients were identified. Three patients under the age of 18 (1 NUT-sarcoma and 2 rhabdomyosarcoma) were excluded from the analysis, as were 9 patients with other types of sarcoma and 5 with hemangiopericytoma. Accordingly, 226 patients were analysed. Demographics, site, stage, histopathology, and treatment data are presented in Table 1. SCC was the most common subtype (53.1\%), followed by SNMM (12.8\%), adenocarcinoma (10.2\%), salivary carcinoma (8.8\%), ENB (6.6\%), SNUC (5.8\%), SNEC (2.2\%), and NUT (0.4\%). Eighty percent completed treatment with curative intent. The treatment consisted of multimodality regimes including surgery with neoadjuvant $(29 \%)$ or adjuvant $(37 \%) \mathrm{RT} \pm \mathrm{CT}$, as well as monotherapy regimes with surgery $(22 \%)$, definitive 
Table 1 Demographics, tumor characteristics, treatment intention, and outcome by histologic subtype for 226 patients with sinonasal malignancies

\begin{tabular}{|c|c|c|c|c|c|c|c|c|c|}
\hline & $\begin{array}{l}\text { All } \\
(n=226)\end{array}$ & $\begin{array}{l}\text { SCC } \\
(n=120)\end{array}$ & $\begin{array}{l}\text { SNMM } \\
(n=29)\end{array}$ & $\begin{array}{l}\text { Adeno } \\
(n=23)\end{array}$ & $\begin{array}{l}\text { Salivary }^{\mathrm{a}} \\
(n=20)\end{array}$ & $\begin{array}{l}\text { ENB } \\
(n=15)\end{array}$ & $\begin{array}{l}\text { SNUC } \\
(n=13)\end{array}$ & $\begin{array}{l}\text { SNEC } \\
(n=5)\end{array}$ & $\begin{array}{l}\text { NUT } \\
(n=1)\end{array}$ \\
\hline \multicolumn{10}{|l|}{ Age } \\
\hline Mean/median & $66.0 / 67$ & $67.5 / 68$ & $68.3 / 69$ & $61.1 / 65$ & $60.6 / 60$ & $62.7 / 67$ & $71.7 / 69$ & $58.8 / 54$ & 40 \\
\hline Range & 27-97 & $40-91$ & $51-84$ & $31-83$ & 30-93 & $41-78$ & $52-97$ & $27-90$ & 40 \\
\hline Sex (male) $(\%)$ & 57 & 68 & 45 & 52 & 40 & 53 & 39 & 40 & 0 \\
\hline \multicolumn{10}{|l|}{$\operatorname{Stage}^{\mathrm{b}}(\%)$} \\
\hline I & 16 & 24 & NA & 13 & 10 & 13 & & 20 & \\
\hline II & 12 & 9 & NA & 30 & 30 & 13 & & & \\
\hline III & 11 & 14 & 14 & 9 & 5 & & & & \\
\hline IVA & 31 & 28 & 69 & 26 & 20 & 27 & 23 & & \\
\hline IVB & 26 & 22 & 10 & 22 & 30 & 47 & 69 & 60 & \\
\hline IVC & 4 & 3 & 7 & & 5 & & 8 & 20 & 100 \\
\hline \multicolumn{10}{|l|}{ Primary site (\%) } \\
\hline Nasal cavity & 58 & 54 & 72 & 57 & 65 & 73 & 31 & 60 & \\
\hline Maxillary & 22 & 29 & 10 & 22 & 20 & & 8 & & 100 \\
\hline Ethmoid & 3 & 3 & 3 & 13 & & & & & \\
\hline Frontal & 0 & 1 & & & & & & & \\
\hline Sphenoid & 4 & 4 & 3 & & 5 & & 8 & & \\
\hline Overlapping $^{\mathrm{c}}$ & 14 & 9 & 10 & 9 & 10 & 27 & 54 & 40 & \\
\hline \multicolumn{10}{|c|}{ Curative treatment intention (\%) } \\
\hline Initiated & 84 & 83 & 93 & 96 & 70 & 100 & 69 & 60 & 0 \\
\hline Completed & 80 & 80 & 76 & 96 & 70 & 100 & 62 & 60 & 0 \\
\hline \multicolumn{10}{|l|}{ Survival (months) } \\
\hline Mean $\mathrm{OS} \pm \mathrm{SD}$ & $88.7 \pm 5.6$ & $77.7 \pm 6.7$ & $69.2 \pm 10.9$ & $120.6 \pm 15.8$ & $103.1 \pm 19.2$ & $135.1 \pm 9.6$ & $50.5 \pm 12.6$ & $29.4 \pm 13.9$ & 5 \\
\hline Median OS & 76 & 51 & 69 & 117 & 88 & 147 & 42 & 9 & 5 \\
\hline $95 \% \mathrm{CI}$ & $57-94$ & $20-82$ & $28-110$ & 89-145 & $26-150$ & $56-238$ & $0-86$ & $5-13$ & \\
\hline 5 -year OS (\%) & 56.6 & 48.1 & 54.9 & 77.3 & 70.0 & 100 & 44.0 & 20.0 & 0 \\
\hline 10 -year OS (\%) & 35.1 & 34.6 & 29.1 & 48.9 & 46.7 & 79.5 & 0 & 0 & \\
\hline
\end{tabular}

SCC squamous cell carcinoma, SNMM sinonasal mucosal melanoma, Adeno adenocarcinoma, Salivary salivary carcinoma, ENB esthesioneuroblastoma, SNUC sinonasal undifferentiated carcinoma, SNEC sinonasal neuroendocrine carcinoma, NUT NUT carcinoma

${ }^{a}$ The histology for the 20 patients diagnosed with salivary carcinoma was adenoid cystic cancer $(n=13)$, salivary duct carcinoma ( $\left.n=2\right)$, epithelial-myoepithelial carcinoma $(n=2)$, acinic cell cancer $(n=1)$, mucoepidermoid cancer $(n=1)$, and cancer ex pleomorphic adenoma $(n=1)$. The 5 -year OS rate for patients with adenoid cystic cancer was $76.9 \%$ (median OS was 86 months)

${ }^{\mathrm{b}}$ Stage according to UICC TNM 8th edition

${ }^{\mathrm{c}}$ Overlapping lesion of accessory sinuses (C31.8)

RT + CT (7\%), or RT (5\%). Follow-up was 100\%. The median follow-up time was 106 (95\% CI 94-119) months.

\section{Overall survival}

Median OS for all patients was 76 (95\% CI 57-94) months with 5- and 10-year OS rates of 57\% and 35\%, respectively. Patients with ENB and adenocarcinoma had best prognosis, salivary carcinoma intermediate prognosis, whereas other histopathologies had poorer prognosis (Fig. 1a). The median OS by stage was 125 months for stage I $(n=37)$, 146 for stage II $(n=26), 140$ for stage III $(n=25), 94$ for stage IVA $(n=69), 15$ for stage IVB $(n=59)$, and 7 for stage
IV C $(n=10)$ (Fig. 1b). Patients with tumors located in the nasal cavity had significantly better survival compared to other subsites (Fig. 1c). The median OS by subsite was 109 months for nasal cavity, 71 for ethmoid sinus, 29 for maxillary sinus, 37 for overlapping lesions, 15 for frontal, and 9 for sphenoid sinus. As indicated in Fig. 1d, OS also varied significantly with treatment intent and modality $(p<0.001)$.

The results of the univariable and multivariable analyses for OS (risk for death) are listed in Table 2. Age, T and $\mathrm{N}$-classification, site, stage, histopathology, and treatment were all significant risk factors $(p<0.001)$. Increasing age, stage IVA disease or higher, paranasal tumor localization, 

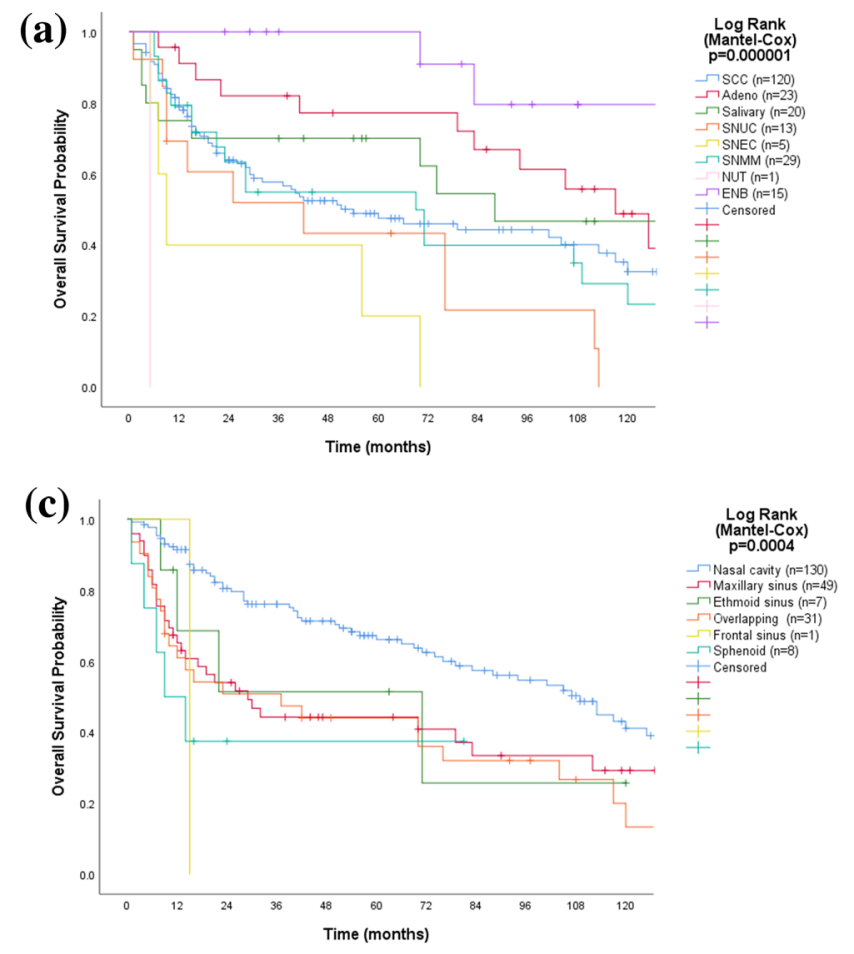

Fig. 1 Kaplan-Meier curves displaying overall survival by histopathology (a), stage (b), site (c), and treatment (d) for 226 patients with SNM. SCC squamous cell carcinoma, Adeno adenocarcinoma, Salivary salivary carcinoma, SNUC sinonasal undifferentiated carci-

NUT histopathology, and treatment with palliative intention remained as significant independent negative prognostic factors in the multivariable analysis. There were no significant differences in survival between patients treated in the early (2001-2009) and the latter half (2010-2019) of the study period.

\section{Treatment with curative intent}

Treatment with curative intent was initiated in $84 \%$ of the 226 patients but could only be completed in $80 \%$. Demographics, tumor characteristics, treatment, and outcome for the 180 patients who completed curative treatment are depicted in Table 3. Of these patients, $158(88 \%)$ patients had surgery, 141 (78\%) had RT, and 37 (21\%) CT or combinations thereof. Monotherapy was administered to $44 \%$, with only surgery to $22 \%$, definitive chemoradiotherapy $(\mathrm{RT}+\mathrm{CT})$ to $7 \%$, and RT to $5 \%$. Multimodality treatment that included surgery and $\mathrm{RT} \pm \mathrm{CT}$ was administered to $66 \%$. Furthermore, 52 patients $(29 \%)$ received neoadjuvant oncological therapy, whereof 37 had neoadjuvant RT and 15 had neoadjuvant RT +CT. Sixty-six patients (37\%) received adjuvant oncological therapy, whereof 57 had adjuvant RT and 9 had RT + CT. No patient was subjected to neoadjuvant chemotherapy alone. Treatment varied significantly
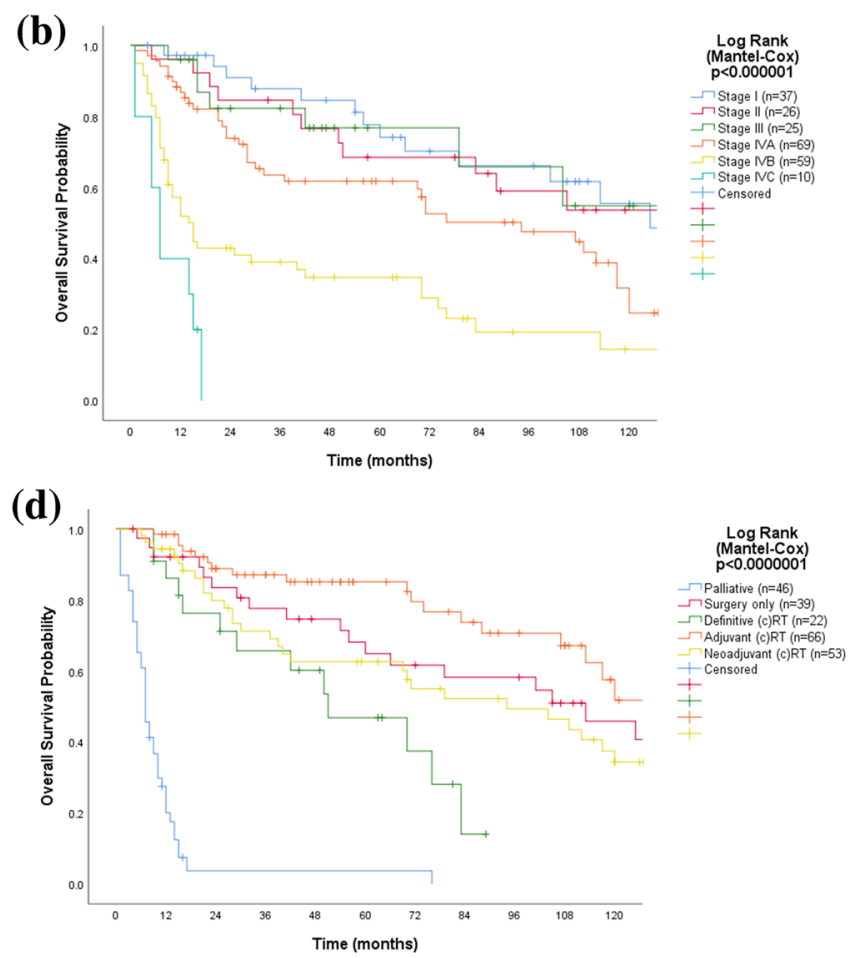

noma, SNEC sinonasal neuroendocrine carcinoma, SNMM sinonasal mucosal melanoma, ENB esthesioneuroblastoma, NUT NUT-carcinoma, $R T \pm C T$ radiotherapy with or without concomitant chemotherapy

according to histology (Table 3$)$ and stage $(p<0.001)$. Most stage I patients had only surgery (73\%), most stage II and III surgery and adjuvant $\mathrm{RT} \pm \mathrm{CT}(50 \%$ and $56 \%$, respectively), most stage IVA neoadjuvant (58\%) or adjuvant (37\%) $\mathrm{RT} \pm \mathrm{CT}$ in combination with surgery, and most stage IVB definitive $\mathrm{RT} \pm \mathrm{CT}(52 \%)$ or surgery with adjuvant $\mathrm{RT} \pm \mathrm{CT}$ $(33 \%)$. The surgery was endoscopic in $19 \%$ and open transfacial in the remaining cases. It included medial maxillectomies in $44 \%$, hemi maxillectomies in $25 \%$, nose amputations in $8 \%$, craniofacial resections in $6 \%$, orbital eviscerations in $6 \%$, and reconstruction with microvascular free flaps in $4 \%$.

\section{Failures and disease-free survival}

During follow-up, $31 \%(n=55)$ of the 180 patients treated with curative intent suffered from recurrences (i.e., 17 local, 15 regional, 17 distant, 2 loco-regional, 2 regio-distant, and 2 all sites). Six patients were never in remission: one stage IVA SCC and 5 stage IVB (2 SCC, 1 SNMM, 1 SNUC, 1 SNEC). The DFS varied significantly according to histopathology $(p=0.022)$, stage $(p=0.0002)$, and treatment modality $(p=0.020)$, but not according to primary site $(p=0.056)$ (Fig. 2). SCC, adenocarcinoma, and ENB had the longest DFS, whereas patients with SNMM, SNEC, and SNUC had the shortest (Table 3). Patients who only had surgery $(n=39)$ 
Table 2 Overall survival and risk for death in 226 patients with sinonasal malignancies

\begin{tabular}{|c|c|c|c|c|c|c|c|c|}
\hline & \multirow[t]{2}{*}{$n$} & \multirow[t]{2}{*}{ Events } & \multicolumn{3}{|c|}{ Univariable Cox } & \multicolumn{3}{|c|}{ Multivariable Cox } \\
\hline & & & $p$ value & HR & $(95 \% \mathrm{CI})$ & $p$ value & HR & $(95 \% \mathrm{CI})$ \\
\hline All patients & 226 & 126 & & & & & & \\
\hline Age & & & & & & 0.002 & 1.03 & $1.01-1.04$ \\
\hline $0-65$ & 99 & 43 & & 1 (Ref.) & & NA & & \\
\hline$>65$ & 127 & 83 & 0.000 & 2.2 & $1.5-3.1$ & & & \\
\hline Sex & & & & & & NA & & \\
\hline Male & 129 & 73 & & 1 (Ref.) & & & & \\
\hline Female & 97 & 53 & 0.404 & 0.9 & $0.6-1.2$ & & & \\
\hline $\mathrm{T}$ classification & & & & & & NA & & \\
\hline $\mathrm{T} 1$ & 38 & 15 & 0.000 & 1 (Ref.) & & & & \\
\hline $\mathrm{T} 2$ & 29 & 14 & 0.680 & 1.2 & $0.6-2.4$ & & & \\
\hline $\mathrm{T} 3$ & 23 & 6 & 0.668 & 0.8 & $0.3-2.1$ & & & \\
\hline $\mathrm{T} 4 \mathrm{a}$ & 68 & 37 & 0.051 & 1.8 & $1.0-3.3$ & & & \\
\hline $\mathrm{T} 4 \mathrm{~b}$ & 68 & 54 & 0.000 & 4.3 & $2.4-7.6$ & & & \\
\hline \multicolumn{9}{|l|}{$\mathrm{N}$ classification } \\
\hline N0 & 204 & 110 & & 1 (Ref.) & & NA & & \\
\hline $\mathrm{N}+$ & 22 & 16 & 0.000 & 2.6 & $1.6-4.5$ & & & \\
\hline \multicolumn{9}{|l|}{ Stage } \\
\hline I & 37 & 14 & 0.000 & 1 (Ref.) & & 0.001 & 1 (Ref.) & \\
\hline II & 26 & 12 & 0.814 & 1.1 & $0.5-2.4$ & 0.181 & 1.9 & $0.7-4.7$ \\
\hline III & 25 & 8 & 0.772 & 1.1 & $0.5-2.7$ & 0.402 & 1.5 & $0.6-4.0$ \\
\hline IVA & 69 & 38 & 0.037 & 1.9 & $1.04-3.6$ & 0.028 & 2.7 & $1.1-6.8$ \\
\hline IVB & 59 & 45 & 0.000 & 4.5 & $2.4-8.2$ & 0.000 & 6.9 & $2.6-18.0$ \\
\hline IVC & 10 & 9 & 0.000 & 13.6 & $5.7-32.8$ & 0.031 & 4.0 & $1.1-13.8$ \\
\hline \multicolumn{9}{|l|}{ Tumor site } \\
\hline Nasal cavity & 128 & 61 & & 1 (Ref.) & & & 1 (Ref.) & \\
\hline Paranasal & 98 & 65 & 0.000 & 2.0 & $1.4-2.8$ & 0.002 & 1.03 & $1.01-1.04$ \\
\hline \multicolumn{9}{|l|}{ Histopathology } \\
\hline SCC & 120 & 65 & 0.000 & 1 (Ref.) & & 0.005 & 1 (Ref.) & \\
\hline Adeno & 23 & 11 & 0.052 & 0.5 & $0.3-1.0$ & 0.080 & 0.5 & $0.3-1.1$ \\
\hline Salivary & 20 & 10 & 0.325 & 0.7 & $0.4-1.4$ & 0.263 & 0.7 & $0.3-1.4$ \\
\hline SNUC & 13 & 11 & 0.084 & 1.8 & $0.9-3.3$ & 0.012 & 0.4 & $0.2-0.8$ \\
\hline SNEC & 5 & 5 & 0.019 & 3.0 & $1.2-7.5$ & 0.187 & 1.9 & $0.7-4.9$ \\
\hline SNMM & 29 & 20 & 0.568 & 1.2 & $0.7-1.9$ & 0.664 & 0.9 & $0.5-1.6$ \\
\hline NUT & 1 & 1 & 0.012 & 13.4 & $1.8-102$ & 0.049 & 9.1 & $1.0-82.3$ \\
\hline ENB & 15 & 3 & 0.011 & 0.2 & $0.1-0.7$ & 0.007 & 0.2 & $0.1-0.6$ \\
\hline \multicolumn{9}{|l|}{ Treatment } \\
\hline Surgery only & 39 & 21 & 0.000 & 1 (Ref.) & & 0.000 & 1 (Ref.) & \\
\hline Multimodality & 119 & 48 & 0.421 & 0.8 & $0.5-1.4$ & 0.145 & 0.6 & $0.3-1.2$ \\
\hline Definitive $\mathrm{RT} \pm \mathrm{CT}$ & 22 & 14 & 0.020 & 2.3 & $1.1-4-6$ & 0.478 & 0.7 & $0.2-1.9$ \\
\hline Palliative & 46 & 43 & 0.000 & 17.2 & $9.3-31.7$ & 0.001 & 5.4 & $2.0-14.3$ \\
\hline
\end{tabular}

Age (binary variable lit at 65 years) as well as $\mathrm{T}$ and $\mathrm{N}$ classification were pre-selected to be included in univariate analysis, whereas age as a continuous variable and stage were pre-selected to be included in multivariable model

$H R$ hazard ratios from uni- and multivariable Cox regression, $N A$ not applicable, Ref. reference, $R T \pm C T$ chemoradiotherapy that included chemotherapy in $7 \%$ of the cases, SCC squamous cell carcinoma, SNMM Sinonasal mucosal melanoma, Adeno adenocarcinoma, Salivary salivary carcinoma, ENB esthesioneuroblastoma, SNUC sinonasal undifferentiated carcinoma, SNEC sinonasal neuroendocrine carcinoma, NUT NUT carcinoma, $R T \pm C T$ radiotherapy with or without concomitant chemotherapy 


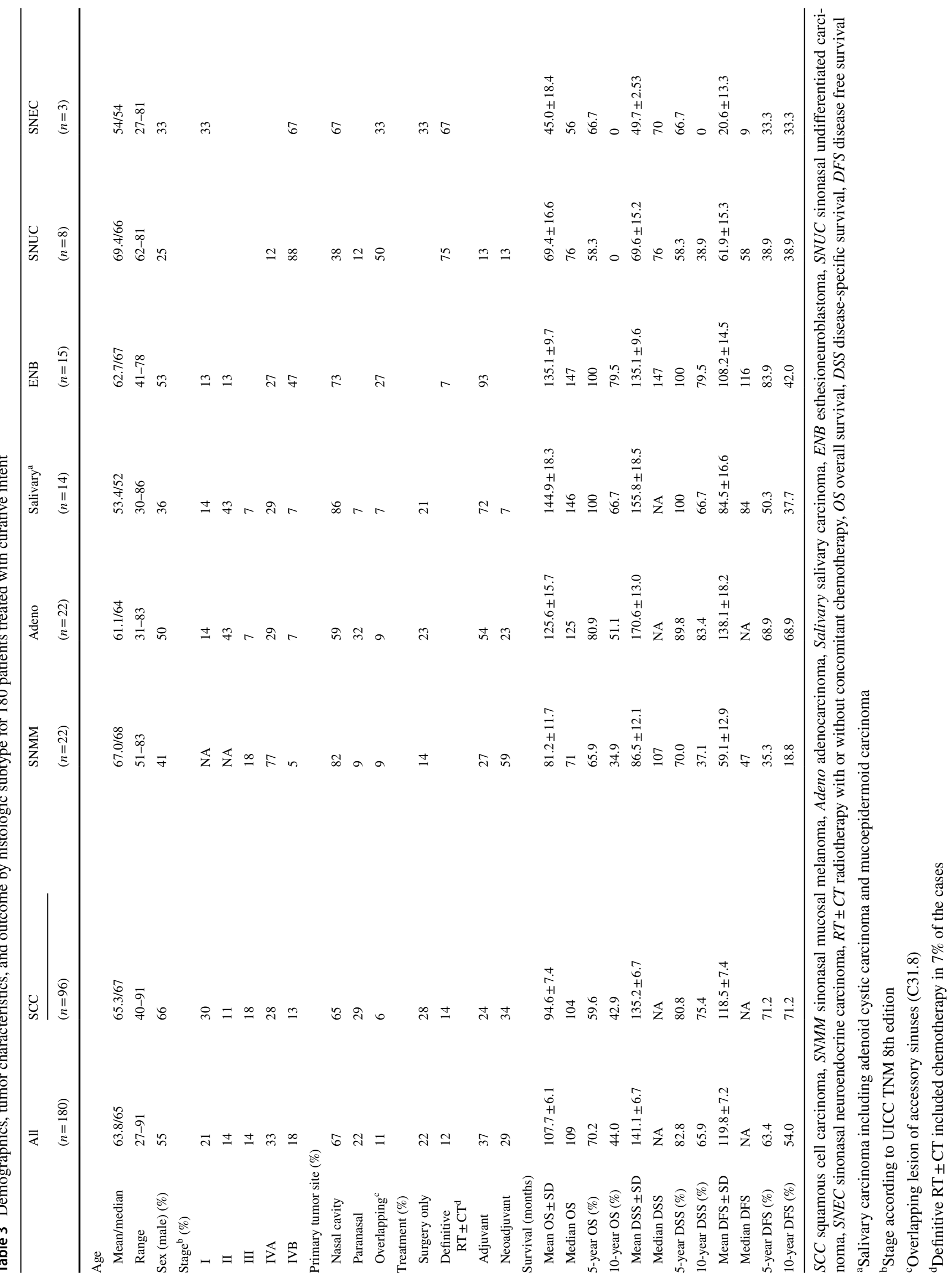



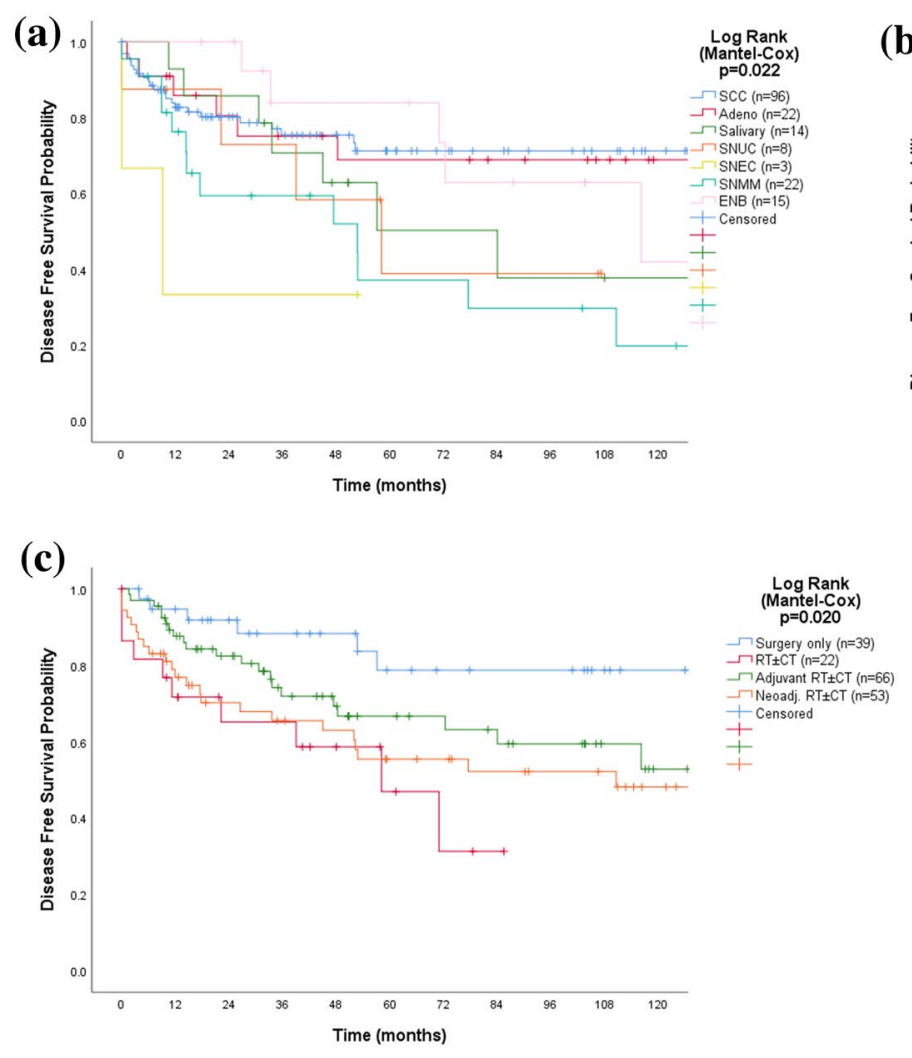

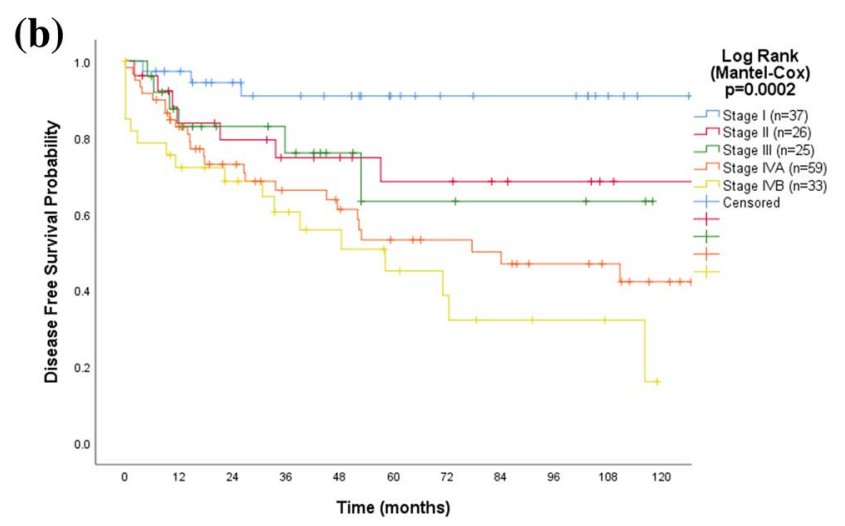

Fig. 2 Kaplan-Meier curves displaying disease free survival by histopathology (a), stage (b), and treatment (c) for 180 patients with SNM treated with curative intent. SCC squamous cell carcinoma, Adeno adenocarcinoma, Salivary salivary carcinoma, SNUC sinonasal undif-

had the longest DFS (mean 119.4 \pm 9.3 months), whereas the 22 patients who received definitive $\mathrm{RT} \pm \mathrm{CT}$ (whereof 13 in combination with concomitant CT) had mean DFS of $49.8 \pm 8.0$ months. Patients who received multimodality treatment with RT $\pm \mathrm{CT}$ in a neoadjuvant setting $(n=53)$ had a mean DFS of $107.8 \pm 12.7$ months and 105.2 \pm 9.3 months in the adjuvant setting $(n=66)$.

T-classification $(p=0.002)$, regional metastases $(p=0.005)$, stage $(p=0.002)$ histopathology $(p=0.020)$, and treatment $(p=0.033)$, but not age or gender had significant impact on risk for recurrences or never being in remission (univariable analysis). According to multivariable regression analysis, stage $(p=0.001)$ and histopathology $(p=0.030)$ remained as significant independent factors for recurrence: stage III (HR 4.6 [95\% CI 1.1-18.4]), stage IVA (HR 5.9 [95\% CI 1.7-20.8]), stage IVB (HR 12.8 [95\% CI 3.6-45.2]), SNEC (HR 5.0 [95\% CI 1.1-23.1]), and melanoma histopathology (HR 2.2 [95\% CI 1.1-4.5]).

\section{Disease-specific survival}

During follow-up, 44 of the 180 patients treated with curative intent succumbed to their disease and 38 died of ferentiated carcinoma, SNEC sinonasal neuroendocrine carcinoma, $S N M M$ sinonasal mucosal melanoma, ENB esthesioneuroblastoma, $R T \pm C T$ radiotherapy with or without concomitant chemotherapy

intercurrent disease. The DSS varied significantly according to stage ( $p=0.0000003)$, histopathology $(p=0.0004)$, treatment modality $(p=0.000002)$, and primary site $(p=0.016)$ (Fig. 3). The 5- and 10-year OS and DSS rates by histopathology are presented in Table 3. Patients with adenocarcinoma and salivary carcinoma had the longest DSS, whereas patients with SNUC and SNEC had the shortest. Patients who only had surgery had the longest DSS (mean $150.5 \pm 5.0$ months), whereas patients who received definitive RT \pm CT had a mean DSS time of $54.9 \pm 6.9$ months. Patients who received multimodality treatment with $\mathrm{RT} \pm \mathrm{CT}$ in the neoadjuvant setting had a mean DSS of $131.8 \pm 12.1$ months, whereas it was $148.9 \pm 10.6$ months in the adjuvant setting $(n=66)$. Increasing age (1-year increments) (HR 1.04 [95\% CI 1.02-1.06]), stage IVB disease (HR 4.6 [95\% CI 2.1-10.0]), melanoma histopathology (HR 4.7 [95\% CI 2.0-10.9]), and treatment with definitive $\mathrm{RT} \pm \mathrm{CT}(p=0.001, \mathrm{HR} 17.3$ [95\% CI 3.3-90.9]) remained as significant independent prognostic factors for disease-specific mortality (DSM) according to multivariable regression analysis $(p \leq 0.001)$. 

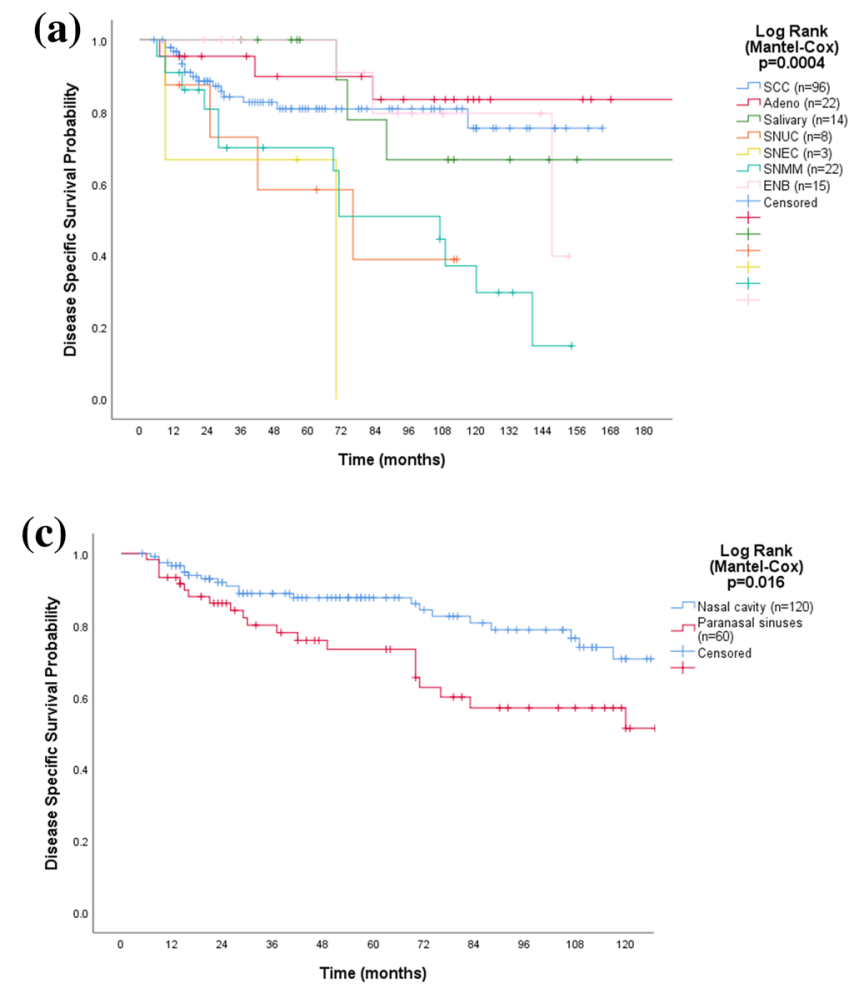

Fig. 3 Kaplan-Meier curves displaying disease-specific survival by histopathology (a), stage (b), site (c), and given treatment (d) for 180 patients with SNM treated with curative intent. SCC squamous cell carcinoma, Adeno adenocarcinoma, Salivary salivary carcinoma,

\section{Stage IVB disease}

Fifty-nine patients in the total cohort were diagnosed with stage IVB disease. During follow-up, 42 succumbed to their malignancy and 3 died of intercurrent disease. The median OS was 15 months. OS varied significantly by histopathology $(p=0.003)$ and was longest for ENB (median 83 months) while shortest for salivary carcinoma (median 4 months), SNMM (median 7 months), and SNEC (median 9 months). Treatment with curative intent was administered to $56 \%(n=33)$ despite that 20 of those patients had intracranial, 12 sphenoidal, and/or 7 nasopharyngeal involvements. Six patients had tumors involving the orbital apex and 5 the retro-maxillary space. Treatment varied significantly according to histopathology $(p=0.005)$. All 33 patients subjected to curative treatment received RT. Seventeen had definitive RT \pm CT $(8 \mathrm{SCC}$, 6 SNUC, 2 SNEC) and 16 had surgery after neoadjuvant $\mathrm{RT} \pm \mathrm{CT}$ (3 SCC, 1 Adeno, 1 SCC) or before adjuvant $\mathrm{RT} \pm \mathrm{CT}$ (6 ENB, 2 Adeno, $1 \mathrm{SCC}, 1$ salivary, 1 SNUC). The $\mathrm{RT} \pm \mathrm{CT}$ included concomitant $\mathrm{CT}$ in 15 cases, i.e., for $65 \%$ in the definitive $\mathrm{RT} \pm \mathrm{CT}$ group, $27 \%$ in the adjuvant, and $20 \%$ in the neoadjuvant group. The surgery was
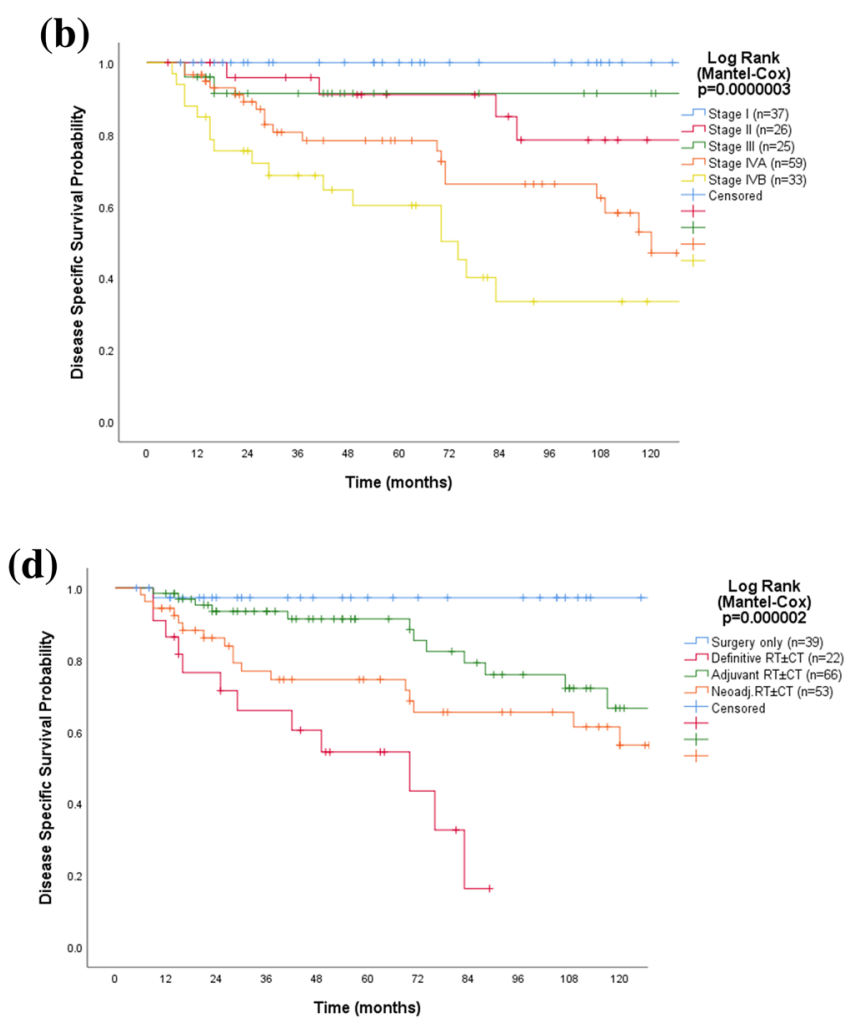

SNUC sinonasal undifferentiated carcinoma, SNEC sinonasal neuroendocrine carcinoma, $S N M M$ sinonasal mucosal melanoma, $E N B$ esthesioneuroblastoma, $R T \pm C T$ radiotherapy with or without concomitant chemotherapy

endoscopic in 9 cases (mostly ENB) and open transfacial in 24. Six patients were never in remission (2 after neoadjuvant treatment and surgery and 4 after definitive $\mathrm{RT} \pm \mathrm{CT}$. During follow-up, 12 additional patients succumbed to their malignancy. The DSS varied significantly according to histopathology $(p=0.00002)$ and was longest for adenocarcinoma and ENB while shortest for SNMM and SNEC (Fig. 4a). Survival also varied significantly according to treatment modality $(p=0.016)$. The 5 -year DSS was $100 \%$ after adjuvant RT $\pm \mathrm{CT}$, whereas it was $40 \%$ after neoadjuvant $\mathrm{RT} \pm \mathrm{CT}$ plus surgery and $43 \%$ after definitive RT \pm CT (Fig. 4b). Having a tumor deemed as not resectable increased risk for DSM ( $p=0.006$, HR 6.1 [95\% CI 1.7-22.1]). Having surgery and adjuvant RT \pm CT reduced the risk for DSM $c f$. definitive and neoadjuvant $\mathrm{RT} \pm \mathrm{CT}(p=0.015, \mathrm{HR} 0.2$ [95\% CI 0.0-0.7]).

\section{Patients treated with palliative intent}

Treatment with palliative intent was administered to $20 \%$ of the cohort $(n=46)$. The median age was 76 (range 40-97) years and $35 \%$ were women. These patients were significantly older than the patients who completed treatment with 


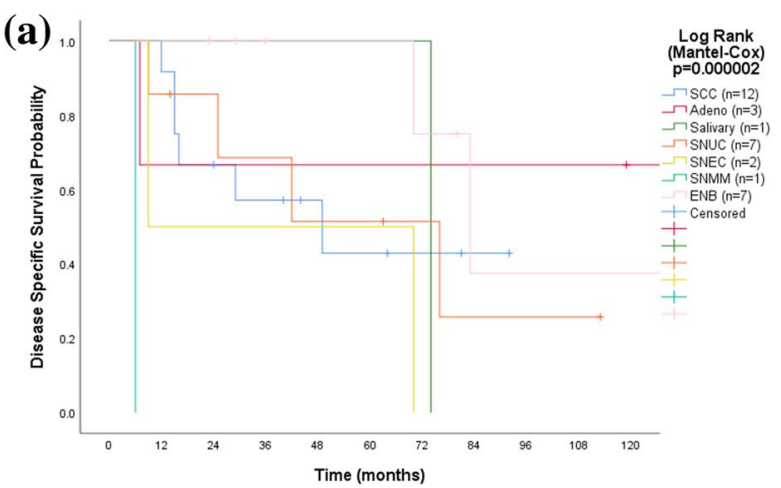

Fig. 4 Kaplan-Meier curves displaying disease-specific survival by histopathology (a) and treatment modality (b) for 33 patients with stage IVB SNM treated with curative intent. SCC squamous cell carcinoma, Adeno adenocarcinoma, Salivary salivary carcinoma, SNUC

curative intent $(p<0.001)$. The histopathology was SCC in $52 \%$, SNMM in $15 \%$, salivary carcinoma in $13 \%$, SNUC in $11 \%$, SNEC in $4 \%$, adenocarcinoma in $2 \%$, and NUTsarcoma in $4 \%$. All presented with stage IV disease (stage IVA $22 \%$, IVB $56 \%$, and IVC 22\%). Best supportive care was administered to all patients, whereof $52 \%$ also received palliative RT, $20 \%$ palliative $\mathrm{RT}+\mathrm{CT}$, and $6 \%$ palliative surgery. The mean survival was $10.3 \pm 2.4$ months. The 1 - and 2 -year OS rates were $28.3 \%$ and $3.9 \%$, respectively.

\section{Discussion}

This study describes characteristics and fate for 226 consecutive patients with de novo SNM, including SNMM and ENB, who were treated at a single tertiary academic referral centre. The lead finding is a seemingly good survival outcome, especially for patients with SNMM, adenocarcinoma, and SNUC. Together with published literature on the matter, our results may provide a background for future RCTs. The strength of our report reflects that it involves a population-based cohort and that curated data have been obtained directly from patient records.

A principal feature of our findings is a seemingly longer survival for the whole cohort (including patients treated with curative and palliative intent) compared to other studies. The 5 -year OS rate of $57 \%$ is numerically better than corresponding rates reported by Thorup et al. (47\%), Koivunen et al. (38\%), Filtenborg et al. (46\%), and Gore et al. (46\%) [4-7]. To the best of our understanding, these discrepancies are probably not explained by factors, such as age, site, or even stage. In fact, a greater percentage of our patients presented with stage IV disease (61\%), $c f$. Thorup (48\%), Koivunen (52\%), and Filtenborg (58\%). Similarly, the apparently better survival in our study $c f$. Gore et al. may not be explained by less advanced tumors, as regional and distant metastases,

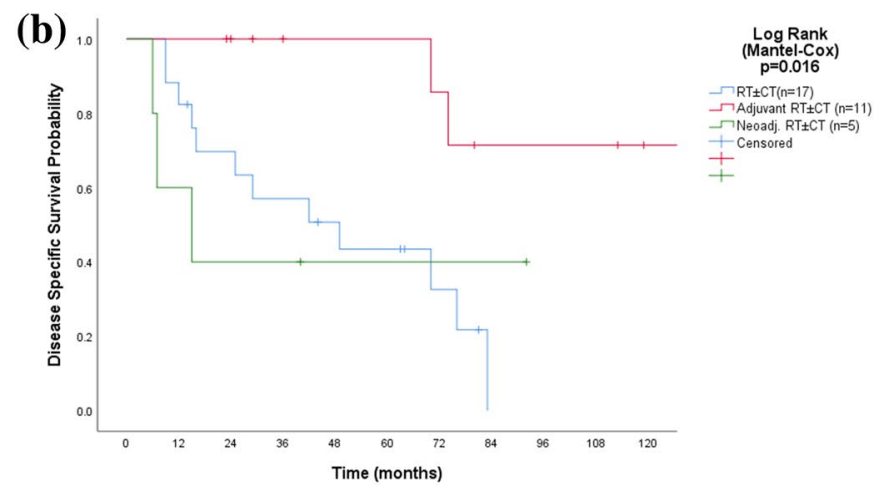

sinonasal undifferentiated carcinoma, SNEC sinonasal neuroendocrine carcinoma, SNMM sinonasal mucosal melanoma, ENB esthesioneuroblastoma, $R T \pm C T$ radiotherapy with or without concomitant chemotherapy

respectively, were present in $9.5 \%$ and $4.1 \%$ in our study cf. in $4.4 \%$ and $1.5 \%$ in the U.S. cohort [7]. Moreover, the 5 -year DSS rate of $83 \%$ for patients treated with curative intent in our cohort is higher than in the two registry-based studies from Denmark reported by Thorup et al. (57\%) and Filtenborg et al. $(56 \%)[4,6]$. However, we fully concede that cohort heterogeneity makes it difficult to reach definitive conclusions regarding survival differences between our study and the Nordic [4-6] and U.S. [2, 7] registry-based reports, i.e., differences in distributions of histopathologies, nasal/paranasal localizations, and tumour stages as well as differences in distributions between treatment with palliative and curative intent.

It is essential to consider the histopathology of SNM in relation to specific outcome measures, because it is an important prognostic factor [8]. Our findings corroborate this, because histopathology influenced DFS, DSS, and OS. Patients with adenocarcinoma and ENB had the best prognosis, while SCC, SNMM, salivary carcinoma, and SNUC had poorer, and SNEC and NUT had the worst prognosis. In 2017, Robin et al. presented survival for SNM per histopathology based on 11,160 patients from of the National Cancer Database, representing $>1,500$ community and academic cancer centres and approximately $70 \%$ of all cases in the U.S. [2]. SCC was most frequent (54\%) followed by SNMM (10\%), adenocarcinoma (7\%), adenoid cystic cancer (7\%), SNUC (4\%), and ENB (10\%). These percentages are similar to what we observed, and heterogeneity in histopathology may not explain the differences in outcomes between the reports. Robin et al. reported no survival rates but instead median OS times: 52.8 months for SCC (cf. 54 months in our cohort), 22.4 months for SNMM ( $c f$. 69 months in our cohort), 98.6 months for adenocarcinoma (cf. 117 months in our cohort), 86.1 months for adenoid cystic cancer ( $c f .86$ months in our cohort), and 33.4 months for SNUC ( $c f .42$ months in our cohort). In the study by 
Robin et al., median OS was not reached for ENB, whereas it was 147 months in our cohort.

Survival for patients with SNM of specific histopathologies in the present study seems to surpass what was reported by Robin et al. [2]. The most pronounced difference concerns SNMM. As indicated above, we observed 46 months longer median survival time for SNMM patients than presented by Robin et al. [2]. Moreover, the observed 5-year OS rate of 55\% for all SNMM in the present study agrees with and exceeds the OS previously reported by our group (that included a part of the present study population) [9]. The 55\% OS rate also compares favourably to other studies that generally have indicated 5-year OS rates of less than $40 \%$ for SNMM [7, 10-14]. As discussed in our earlier publication [9], the differences might reflect our frequent utilization of neoadjuvant hyper-fractionated RT with concomitant chemotherapy or adjuvant RT following surgery for stage IVA SNMM. In agreement, recent systematic meta-analyses indicates that adjuvant RT prolongs survival for patients with SNMM [15, 16], while others suggest adjuvant RT does not prolong survival yet increases local control [17]. From the limited number of 17 patients with stage IVA SNMM in this study, the only significant difference that emerged in the context of neoadjuvant/adjuvant measures was that patients subjected to RT + CT $(n=12)$ had a longer OS (mean $81.9 \pm 13.2$, median 71 months) than those who only received RT $(n=5)$ in addition to the surgery (mean $22.5 \pm 2.9$, median 21 months) $(p=0.046)$. According to multivariable regression analysis, treatment with $\mathrm{RT} \pm \mathrm{CT} v s$. RT remained as a significant independent prognostic factor for survival reducing the risk of death for stage IVA patients (HR 0.16 [95\% CI 0.029-0.924]) after adjusting for age, and neoadjuvant or adjuvant treatment regimens $(p=0.040)$. Taken together, our observations suggest the possibility that patients with advanced SNMM may benefit from concomitant RT + CT before surgery, but of course RCTs focusing on adjuvant therapies are warranted.

Despite recent therapeutic advances, the prognosis for SNUC remains poor [18]. One of our observations was a seemingly good outcome for patients with SNUC with reservation for the small subset of eight patients. The median OS was 42 months compared to 33 months reported by Robin et al. [2]. Multimodal therapy is a well-established approach for treating SNUC [19]. For example, in a cohort of 95 patients with SNUC who received induction chemotherapy ahead of definitive therapy, 5-year DSS was 59\% [19]. The 5-year DSS of 58\% was similar in our cohort of SNUC-patients subjected to treatment with curative intent. However, only two patients received concomitant RT + CT and the other six RT (combined with surgery in two cases). This outcome corroborates findings by Lehrich et al., in a study involving 440 patients, indicating that induction chemotherapy may not provide survival benefits in SNUC patients
[20]. As for many other subsets of SNM, further studies are warranted also for SNUC. Arguably, such may involve selecting patients for definitive treatment based on response to chemotherapy (a.k.a. chemoselection), as suggested by Amit et al. [19], or based on overall immune phenotype, as suggested for other malignancies [21]. Another conspicuous finding in our study was the long survival for patients with adenocarcinoma $(n=23)$. The median OS was 18 months longer than presented by Robin et al. [2]. We report DSSrates of $89 \%$ and $83 \%$ as well as OS-rates of $77 \%$ and $49 \%$ after 5 and 10 years, respectively. These rates can be compared to a Norwegian cohort $(n=20)$ with DSS-rates of $73 \%$ and $58 \%$ and OS-rates of $68 \%$ and $54 \%$ after 5 and 10 years, respectively [22]. Again, RCTs are warranted stratified per histology, site and stage.

Despite that ENB was described already in 1924, and given the rarity of the malignancy (3-6\% of all SNM), no consensus has been reached regarding staging and optimal treatment strategy [23]. The most widely accepted, yet unofficial, classification for ENB was proposed by Kadish et al. in 1976 [24]. However, other proposed staging systems include the conventional TNM staging by the UICC as well as a modified version of the TNM classification proposed by Dulguerov in 1992 [23, 25]. We chose to use the UICC TNM system to better be able to compare the outcome for ENB with other histopathologies. We observed an apparent high survival for ENB. Both the DSS and OS rates were $100 \%$ at 5 years and $79.5 \%$ at 10 years. In comparison, Jethanamest et al. presented much lower OS rates $(62.1 \%$ at 5 years and $45.6 \%$ at 10 years) in a cohort of 311 ENB patients despite that the mean age was lower than in our cohort (mean age $54 c f .63$ years) [26]. Moreover, in another cohort of 26 ENB patients, $74 \%$ were alive at 5 years and $60 \%$ at 10 years [25]. The apparent better outcome in our cohort may be a coincidence due to comparatively few patients. Another explanation may be that all ENB patients, except one who declined surgery, received multimodality therapy with surgery and adjuvant $\mathrm{RT} \pm \mathrm{CT}$.

Stage IVB disease infers a dismal prognosis and stage IVB patients are often subjected to aggressive treatment. Therefore, conscientious considerations of treatment sequelae and survival probability are essential. In our cohort, $26 \%$ were diagnosed with stage IVB disease. More than half of these patients were treated with curative intent that included RT in all cases and surgery in almost half. A tumor not considered resectable significantly increased the risk for DSM. Accordingly, this meant a treatment regimen that did not include surgery. A couple considerations may be made in this context. First, there are histopathological subsets of stage IVB disease where surgery seems to be futile, e.g., SNMM. Second, there may be other subsets, e.g., ENB, salivary carcinoma, and adenocarcinoma, where a gain by surgery if combined with adjuvant therapy may well offset 
any morbidity. Indeed, the 5-year DSS was $100 \%$ for stage IVB patients who received adjuvant $\mathrm{RT} \pm \mathrm{CT} c f .43 \%$ after definitive $\mathrm{RT} \pm \mathrm{CT}$ and $40 \%$ after neoadjuvant $\mathrm{RT} \pm \mathrm{CT}$, suggesting a benefit of multimodality treatment regime of surgery with adjuvant $\mathrm{RT} \pm \mathrm{CT}$. This was confirmed through the multivariable regression analysis, implying that adjuvant $\mathrm{RT} \pm \mathrm{CT}$ significantly reduced the risk for DSM ( $c f$. definitive and neoadjuvant $\mathrm{RT} \pm \mathrm{CT}$ ). However, except for resectabilty, no other factors, such as age, gender, site, stage, histopathology, or chemotherapy affected risk for DSM. In this study, $19 \%$ of the surgical procedures were endoscopic and the remainder open. In part this may reflect a development over time, with open surgery being standard and endoscopic surgery becoming more common, but sometimes also a deliberate strategy to avoid any piecemeal resection of the tumour. However, the numbers in this study are too low to allow for a comparison between endoscopic and open approaches stratified per histology, site, and stage. To the best of our understanding, there are no RCTs comparing aspects of survival between two surgical approaches for SNM, but such studies are warranted.

In conclusion, our results indicate a seemingly good outcome for SNM patients compared to previous reports, particularly for SNMM, adenocarcinoma, and SNUC. The strength is that the study is population-based and involves consecutive patients assessed and treated at a single tertiary academic referral centre. On the other hand, a weakness is that the material is not stratified for histological margin status. However, such an analysis is very difficult to perform retrospectively, since the question of microscopic radicality and tumour margins frequently involves a close discussion between the pathologist and the surgeon even for en bloc resections. Nevertheless, our observations emphasize the importance of histopathology as a risk factor for SNM and indicate a need for multi-centre RCTs stratified per histopathology and possibly involving multimodality strategies.

Funding Open access funding provided by Lund University.

Open Access This article is licensed under a Creative Commons Attribution 4.0 International License, which permits use, sharing, adaptation, distribution and reproduction in any medium or format, as long as you give appropriate credit to the original author(s) and the source, provide a link to the Creative Commons licence, and indicate if changes were made. The images or other third party material in this article are included in the article's Creative Commons licence, unless indicated otherwise in a credit line to the material. If material is not included in the article's Creative Commons licence and your intended use is not permitted by statutory regulation or exceeds the permitted use, you will need to obtain permission directly from the copyright holder. To view a copy of this licence, visit http://creativecommons.org/licenses/by/4.0/.

\section{References}

1. Stelow EB, Bishop JA (2017) Update from the 4th Edition of the World Health Organization Classification of Head and Neck Tumours: tumors of the Nasal Cavity, Paranasal Sinuses and Skull Base. Head Neck Pathol 11(1):3-15. https://doi.org/10. 1007/s12105-017-0791-4 (PubMed PMID: 28247233; PubMed Central PMCID: PMCPMC5340732)

2. Robin TP, Jones BL, Gordon OM, Phan A, Abbott D, McDermott JD et al (2017) A comprehensive comparative analysis of treatment modalities for sinonasal malignancies. Cancer 123(16):3040-3049. https://doi.org/10.1002/cncr.30686 (PubMed PMID:28369832;PubMedCentralPMCID:PMCPMC6234843)

3. Van Dijk BA, Gatta G, Capocaccia R, Pierannunzio D, Strojan P, Licitra L et al (2012) Rare cancers of the head and neck area in Europe. Eur J Cancer 48(6):783-796. https://doi.org/10.1016/j. ejca.2011.08.021 (PubMed PMID: 22051735)

4. Thorup C, Sebbesen L, Dano H, Leetmaa M, Andersen M, Buchwald $C$ et al (2010) Carcinoma of the nasal cavity and paranasal sinuses in Denmark 1995-2004. Acta Oncol 49(3):389-394. https://doi.org/10.3109/02841860903428176 (PubMed PMID: 20001493)

5. Koivunen P, Makitie AA, Back L, Pukkila M, Laranne J, Kinnunen I et al (2012) A national series of 244 sinonasal cancers in Finland in 1990-2004. Eur Arch Otorhinolaryngol 269(2):615621. https://doi.org/10.1007/s00405-011-1615-x (PubMed PMID: 21590483)

6. Filtenborg MV, Lilja-Fischer JK, Sharma MB, Primdahl H, Kjems J, Plaschke CC et al (2021) Sinonasal cancer in Denmark 20082015: a population-based phase-4 cohort study from DAHANCA. Acta Oncol 60(3):333-342. https://doi.org/10.1080/0284186X. 2021.1874618 (PubMed PMID: 33544640)

7. Gore MR (2018) Survival in sinonasal and middle ear malignancies: a population-based study using the SEER 1973-2015 database. BMC Ear Nose Throat Disord 18:13. https://doi.org/10. 1186/s12901-018-0061-4 (PubMed PMID:30116158;PubMed CentralPMCID:PMCPMC6085622)

8. Dulguerov P, Jacobsen MS, Allal AS, Lehmann W, Calcaterra T (2001) Nasal and paranasal sinus carcinoma: are we making progress? A series of 220 patients and a systematic review. Cancer 92(12):3012-3029. https://doi.org/10.1002/1097-0142(20011215) 92:12\%3c3012::aid-cncr10131\%3e3.0.co;2-e (PubMed PMID: 11753979)

9. Hafstrom A, Brun E, Persson S, Sjovall J, Wahlberg P, Greiff L (2019) Survival benefits from concomitant chemoradiotherapy before radical surgery in stage IVA sinonasal mucosal melanoma? Laryngoscope Investig Otolaryngol 4(6):624-631. https://doi.org/ 10.1002/lio2.317 (PubMedPMID:31890880;PubMedCentralP MCID:PMCPMC6929603)

10. Koivunen P, Back L, Pukkila M, Laranne J, Kinnunen I, Grenman R et al (2012) Accuracy of the current TNM classification in predicting survival in patients with sinonasal mucosal melanoma. Laryngoscope 122(8):1734-1738. https://doi.org/10.1002/lary. 23343 (PubMed PMID: 22549303)

11. Lund VJ, Chisholm EJ, Howard DJ, Wei WI (2012) Sinonasal malignant melanoma: an analysis of 115 cases assessing outcomes of surgery, postoperative radiotherapy and endoscopic resection. Rhinology 50(2):203-210. https://doi.org/10.4193/Rhino11.267 (PubMed PMID: 22616083)

12. Gilain L, Houette A, Montalban A, Mom T, Saroul N (2014) Mucosal melanoma of the nasal cavity and paranasal sinuses. Eur Ann Otorhinolaryngol Head Neck Dis 131(6):365-369. https:// doi.org/10.1016/j.anorl.2013.11.004 (PubMed PMID: 24906226)

13. Lombardi D, Bottazzoli M, Turri-Zanoni M, Raffetti E, Villaret AB, Morassi ML et al (2016) Sinonasal mucosal melanoma: a 
12-year experience of 58 cases. Head Neck 38(Suppl 1):E1737E1745. https://doi.org/10.1002/hed.24309 (PubMed PMID: 26676523)

14 Amit M, Tam S, Abdelmeguid AS, Kupferman ME, Su SY, Raza SM et al (2018) Patterns of treatment failure in patients with sinonasal mucosal melanoma. Ann Surg Oncol 25(6):1723-1729. https://doi.org/10.1245/s10434-018-6465-y (PubMed PMID: 29626308)

15. Hu R, Yang BB (2018) Surgery alone versus post-operative radiotherapy for sinonasal malignant melanoma: a meta-analysis. J Laryngol Otol 132(12):1051-1060. https://doi.org/10.1017/S0022 215118002189 (PubMed PMID: 30674363)

16. Grant-Freemantle MC, Lane O'Neill B, Clover AJP (2021) The effectiveness of radiotherapy in the treatment of head and neck mucosal melanoma: systematic review and meta-analysis. Head Neck 43(1):323-333. https://doi.org/10.1002/hed.26470 (PubMed PMID: 32926490)

17. Jarrom D, Paleri V, Kerawala C, Roques T, Bhide S, Newman L et al (2017) Mucosal melanoma of the upper airways tract mucosal melanoma: a systematic review with meta-analyses of treatment. Head Neck 39(4):819-825. https://doi.org/10.1002/hed.24652 (PubMed PMID: 27898196)

18. Abdelmeguid AS, Bell D, Hanna EY (2019) Sinonasal undifferentiated carcinoma. Curr Oncol Rep 21(3):26. https://doi.org/10. 1007/s11912-019-0776-4 (PubMed PMID: 30806835)

19. Amit M, Abdelmeguid AS, Watcherporn T, Takahashi H, Tam S, Bell D et al (2019) Induction Chemotherapy Response as a Guide for Treatment Optimization in Sinonasal Undifferentiated Carcinoma. J Clin Oncol 37(6):504-512. https://doi.org/10.1200/ JCO.18.00353 (PubMed PMID:30615549;PubMedCentralPM CID:PMCPMC6380524)

20. Lehrich BM, Goshtasbi K, Abiri A, Yasaka T, Sahyouni R, Papagiannopoulos $P$ et al (2020) Impact of induction chemotherapy and socioeconomics on sinonasal undifferentiated carcinoma survival. Int Forum Allergy Rhinol 10(5):679-688. https://doi.org/ 10.1002/alr.22536 (PubMed PMID: 32104985)
21 Nilsson JS, Sobti A, Swoboda S, Erjefalt JS, Forslund O, Lindstedt $\mathrm{M}$ et al (2020) Immune Phenotypes of Nasopharyngeal Cancer. Cancers 12(11):3428. https://doi.org/10.3390/cancers121 13428 (PubMed PMID: 33218184; PubMed Central PMCID: PMCPMC7699205)

22. Konig M, Osnes T, Bratland A, Jebsen P, Meling TR (2020) Treatment of Sinonasal Adenocarcinoma: A Population-Based Prospective Cohort Study. J Neurol Surg B Skull Base 81(6):627-637. https://doi.org/10.1055/s-0039-1694050 (PubMed PMID:3338 1366;PubMedCentralPMCID:PMCPMC7755507)

23. Joshi RR, Husain Q, Roman BR, Cracchiolo J, Yu Y, Tsai J et al (2019) Comparing Kadish, TNM, and the modified Dulguerov staging systems for esthesioneuroblastoma. J Surg Oncol 119(1):130-142. https://doi.org/10.1002/jso.25293 (PubMed PMID:30466166;PubMedCentralPMCID:PMCPMC6352736)

24. Kadish S, Goodman M, Wang CC (1976) Olfactory neuroblastoma. A clinical analysis of 17 cases. Cancer 37(3):1571-1576. https://doi.org/10.1002/1097-0142(197603)37:3<1571::aid-cncr2 820370347>3.0.co;2-1 PubMed PMID: 1260676

25. Dulguerov P, Calcaterra T (1992) Esthesioneuroblastoma: the UCLA experience 1970-1990. Laryngoscope 102(8):843-849. https://doi.org/10.1288/00005537-199208000-00001 (PubMed PMID: 1495347)

26. Jethanamest D, Morris LG, Sikora AG, Kutler DI (2007) Esthesioneuroblastoma: a population-based analysis of survival and prognostic factors. Arch Otolaryngol Head Neck Surg 133(3):276-280. https://doi.org/10.1001/archotol.133.3.276 (PubMed PMID: 17372086)

Publisher's Note Springer Nature remains neutral with regard to jurisdictional claims in published maps and institutional affiliations. 